\title{
COMPROBACIONES RESISTENTES DE ELEMENTOS CONSTRUCTIVOS DE TIERRA
}

\author{
(RESISTANCE CHECKINGS ON EARTH ELEMENTS FOR CONSTRUCTION)
}

\author{
Enrique Estrada, Arquitecto. Erhard Rohmer, Arqto.-Urbanista \\ Equipo Viviendas de Muy Bajo Coste. IETcc-CSIC
}

\section{RESUMEN}

El presente trabajo pretende comparar, por medio de distintos ensayos sobre elementos y fábricas de mamposteria de tierra, las propiedades resistentes obtenidas con las de otros trabajos similares realizados en paises con dilatada tradición y conocimiento de la tierra como material de construcción.

Se han ensayado elementos (adobes y bloques) a compresión y flexotracción y fábricas formadas por conjuntos de los mismos a compresión y a compresión diagonal.

\section{SUMMARY}

This work has te purpose to compare, by means of different tests on adobe bricks and masonry, the obtained resistant properties and those of other similar works carried out in countries having a wide tradition and knowledge about earth as construction material.

Elements (adobe bricks and blocks) have been tested to compression and bending, and masonry made of those elements to compression and diagonal compression.

\section{INTRODUCCION}

Con motivo de la celebración en el IETcc de las jornadas "La Tierra Material de Construcción», organizadas por el equipo «Viviendas de Muy Bajo Coste", se realizaron una serie de ensayos sobre elementos y fábricas de adobe destinados a determinar sus propiedades resistentes, no sólo con carácter absoluto, sino en relación a otros trabajos realizados en Perú y México, en los que además el sismo constituye un peligro cierto y permanente.

El marco de referencia adoptado lo constituyen los resultados de una investigación conjunta (1) llevada a cabo entre el Instituto de Ingenieria de la Universidad Nacional Autónoma de México y el Departamento de Ingenieria de la Pontificia Universidad Católica del Perú, para comparar la resistencia de elementos y mamposteria de adobe hechas en los dos paises y tratar de justificar las importantes diferencias, no fundamentadas, que se presentaban en las resistencias obtenidas en uno y otro pais.

Se pensó que podria complementar este estudio, y aportar un dato más, el ensayar elementos y fábricas en el IETcc según el método reflejado en la Norma Peruana "Construcciones en adobe. Disposiciones especiales para diseño sismorresistente" (2).

Dentro de las diferentes consideraciones acerca de la tierra como material de construcción, destaca, aparte de la argumentación de una resistencia limitada a los agentes atmosféricos, la escasa fiabilidad de las construcciones de tierra frente a los movimientos sismicos.

Aunque esta argumentación, y el propio hecho en si, exige una investigación cada vez más` urgente y detallada, sobre todo teniendo en cuenta que solamente un escaso $5 \%$ de las publicaciones acerca de la tierra tratan de los problemas sismicos, deseamos a priori mencionar algunos criterios para enfocar esta temática bastante compleja sobre la que inciden factores económicos, socio-sicológicos y culturales.

A) La patologia de construcciones de tierra afectadas o dañadas por movimientos sismicos, muestra por encima de todo, y sin entrar en la propia validez de la tierra 


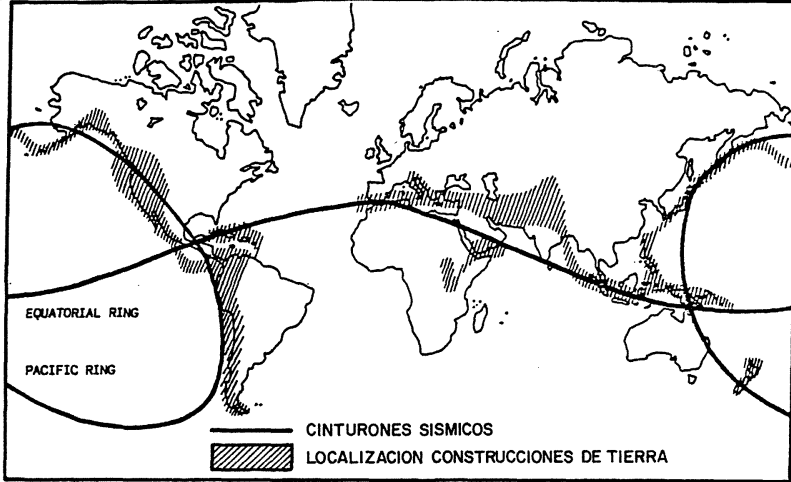

Fig. 1

como material de construcción, defectos de diseño arquitectónico o estructural. No debe olvidarse que mundialmente estas construcciones se encuentran prioritariamente en el ámbito rural, acusando desconocimiento y falta de recursos económicos que impiden la implantación de nuevas pautas de construcción.

B) Las zonas geográficas en las cuales las construcciones de tierra alcanzan un altisimo porcentaje son, por otra parte, las que corresponden a los paises más deprimidos económicamente, y a su vez coinciden con las zonas más afectadas por los movimientos sismicos (Figura 1).

C) Aunque el esfuerzo internacional de ampliar las normas preventivas, y aplicarlas en las zonas rurales y deprimidas está obstaculizado en múltiples ocasiones por una resistencia cultural, falta de medios económicos y de información, en algunos paises como México, Perú y Turquia, entre otros, ya cuentan con normas acerca de los materiales de bajo coste como soluciones alternativas estructurales.

\section{OBJETIVOS}

Como se ha indicado, el objetivo de este trabajo no es otro que mostrar los resultados obtenidos en los distintos ensayos realizados sobre adobes (compresión y flexotracción) y sobre fábricas formadas por conjuntos de los mismos (compresión y compresión diagonal) en relación con los resultados obtenidos con los mismos ensayos en Perú y México.

\section{MATERIALES Y METODOS}

\subsection{Materiales}

Se encuentran a continuación las principales caracteristicas de los distintos tipos de tierra, asi como de los elementos utilizados:
- Tierra Navapalos (Soria), marrón

- Adobes manuales Navapalos, marrón $27 \times 13 \times 10 \mathrm{~cm}$

- Adobes manuales Navapalos, rojo... $27 \times 13 \times 10 \mathrm{~cm}$

- Bloques manuales Navapalos, marrón $38 \times 19 \times 14 \mathrm{~cm}$

- Bloques comprimidos Brepak ........ $29 \times 14 \times 10 \mathrm{~cm}$

Para completar la información véase la revista "Informes de la Construcción», Vol. 37, n.० 377, articulo: “Construcciones en adobe; disposiciones especiales para diseño sismorresistente». NTE E. 080 Adobe; del Instituto Nacional de Investigación y Normalización de la Vivienda de Perú. ININVI.

\subsection{Metodología}

Se han estudiado las siguientes caracteristicas:

- Comportamiento de los suelos (resistencia) en condiciones de laboratorio.

- Resistencia de adobes y bloques a flexotracción y compresión.

- Resistencia a compresión de probetas obtenidas por corte a partir de los adobes y bloques.

- Resistencia a compresión de los diferentes morteros de unión empleados.

- Resistencia a compresión vertical de muretes de esbeltez 3 .

- Resistencia a compresión diagonal (resistencia a corte) de la mamposteria.

\subsection{Confección}

La realización de las probetas $7 \times 7 \times 7 \mathrm{~cm} \mathrm{y}$ $4 \times 4 \times 16 \mathrm{~cm}$ para la comprobación de la resistencia a la compresión y flexotracción de los suelos, se efectuó según el procedimiento descrito en la norma DIN-18952, Hoja 2 (retirada) (3), rompiéndolas después de 28 dias de almacenamiento en un ambiente de $50-60 \%$ de humedad y $170 \mathrm{C}$ con posterior secado en estufa a $60^{\circ} \mathrm{C}$, hasta peso constante.

A los morteros de barro, idénticos a los de los muretes, se añadió aproximadamente un 15\% de arena, para evitar la fisuración y mejorar la trabajabilidad con la paleta. Esta composición cumple los mismos lineamientos que los adobes o bloques; también se añadió aproximadamente un $1 \%$ (en peso) de paja de trigo trillada. Las juntas horizontales $y$ verticales no exceden de $2 \mathrm{~cm}$.

Los morteros de cemento-arena tienen una relación volumétrica de $1 / 7$, utilizando arena gruesa. Las probetas se rompieron igualmente con 28 dias y peso constante. 
La superficie superior se refrentó con $2 \mathrm{~cm}$ de mortero. Para aumentar la adhesión de los morteros a los adobes o bloque se limpió el polvo y se humedecieron antes de realizar las probetas.

\section{RESULTADOS DE LOS ENSAYOS}

\subsection{Comportamiento de los suelos en condiciones de laboratorio}

Se realizaron en laboratorio series de tres probetas cada una, de $7 \times 7 \times 7 \mathrm{~cm}$ y de $4 \times 4 \times 16 \mathrm{~cm}$, con ambos suelos: Navapalos «rojo» y "marrón». En la Lista 1 se recogen los resultados en $\mathrm{kp} / \mathrm{cm}^{2}$ a compresión y flexotracción.

\begin{tabular}{|c|c|c|c|}
\multicolumn{1}{c}{$7 \times 7 \times 7$} & $4 \times 4 \times 16$ & $4 \times 4 \times 16$ \\
k kEKm & 1.1 & 1.2 & 1.3 \\
\hline NAVAPALOS MARRON & 49.25 & 2.2 .33 & 90.83 \\
\hline ROJO & 51.00 & 19.16 & 28.12 \\
\hline
\end{tabular}

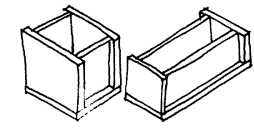

\subsection{Comportamiento de adobes y bloques}

En la Lista 2 se proporcionan los resultados de los cuatro tipos de adobes y bloques (series de tres resultados por cada valor) a flexotracción y cada mitad a compresión. Destaca, como era de esperar, el resultado de los elementos realizados en máquina Brepak, $9,50 \mathrm{kp} / \mathrm{cm}^{2}$ a flexotracción y $22,7 \mathrm{kp} / \mathrm{cm}^{2}$ a compresión.

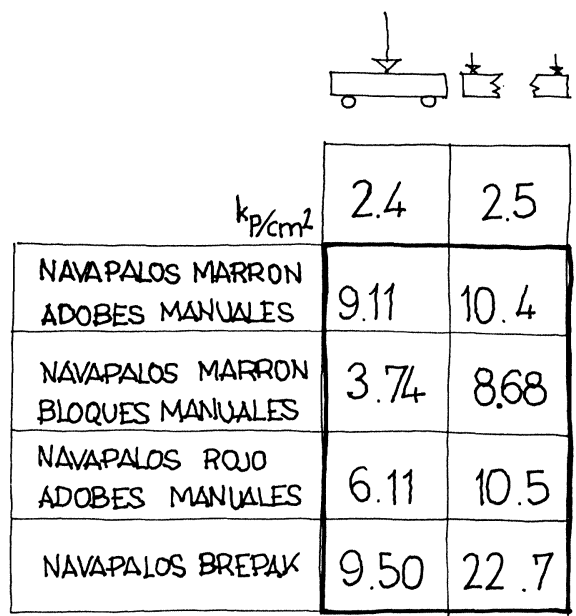

Lista 2

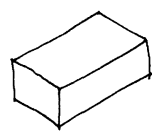

\subsection{Comportamiento de probetas cúbicas}

Las probetas cúbicas, de arista igual al menor de los lados del bloque 0 adobe, se realizaron mediante corte manual y los resultados a compresión se recogen en la Lista 3.

Los dos primeros resultados (Navapalos marrón) son contradictorios con los de la Lista 2, no asi los de Navapalos rojo y Navapalos Brepak, prácticamente coincidentes.

\begin{tabular}{|l|l|}
\multicolumn{1}{c|}{$k_{P} / \mathrm{m}^{2}$} \\
\cline { 2 - 2 } \multicolumn{1}{c|}{} & 3.6 \\
\hline $\begin{array}{l}\text { NAVAPALOS MARRON } \\
\text { CORTADO } 8 \times 8 \times 8\end{array}$ & 36 \\
\hline $\begin{array}{l}\text { NAVAPALOS MARRON } \\
\text { BLOQUE } 14 \times 14 \times 14\end{array}$ & 25 \\
\hline $\begin{array}{l}\text { NAVAPALOS ROJO } \\
\text { CORTADO } 8 \times 8 \times 8\end{array}$ & 10.70 \\
\hline $\begin{array}{l}\text { NAVAPALOS MARRON } \\
\text { BLOQUE } 14 \times 14 \times 14\end{array}$ & 19.31 \\
\hline
\end{tabular}<smiles>CC(=O)C1C2CC3CC(C2)CC1C3</smiles>

Lista 3

\subsection{Comportamiento del material de unión}

De los tres materiales de unión empleados (mortero marrón, mortero rojo y mortero-cemento 1/7) se rompieron a compresión a 28 dias series de probetas de $7 \times 7 \times 7 \mathrm{~cm}$. Los resultados figuran en la Lista 4 .

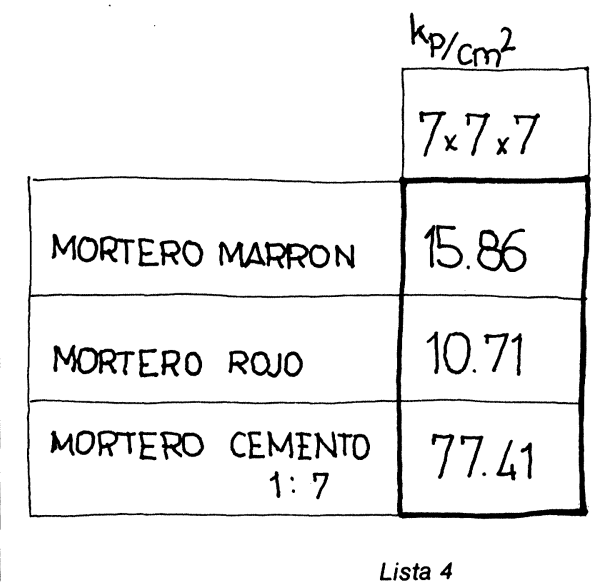


54

Informes de la Construcción, Vol. 38, n.o 385, noviembre, 1986

\subsection{Recopilación de resultados}

En la Tabla 1, se recogen de forma gráfica los resultados comentados en los puntos precedentes.

\section{TABLA 1}

\begin{tabular}{|c|c|c|c|c|c|c|c|c|}
\hline & $\mathrm{kp} / \mathrm{cm}^{2}$ & 1.1 & 1.2 & 1.3 & 2.4 & 2.5 & 3.6 & 4.7 \\
\hline & $\begin{array}{c}\text { SUELO NAVAPANOS } \\
\text { MARRON }\end{array}$ & 49.25 & 22.33 & 90.83 & & & & \\
\hline & $\begin{array}{l}\text { SUELO NAVAPALOS } \\
\text { ROJO }\end{array}$ & 51.0 & 19.16 & 28.12 & & & & \\
\hline & $\begin{array}{l}\text { NAVAPALOS MARPON } \\
\text { ADOBE MANUAL }\end{array}$ & & & & 9.11 & 10.4 & & \\
\hline & $\begin{array}{l}\text { NAVAPALOS MARRON } \\
\text { BLOQUE MANUAL }\end{array}$ & & & & 3.74 & 8.68 & & \\
\hline \% & $\begin{array}{l}\text { NAVAPALOS RONO } \\
\text { ADOBE MANUAL }\end{array}$ & & & & 6.11 & 10.5 & & \\
\hline$\square_{\square}^{\ddagger}$ & $\begin{array}{l}\text { NALLPALOSMARRON } \\
\text { BREPAK }\end{array}$ & & & & 9.50 & 22.7 & & \\
\hline & $\begin{array}{l}\text { NAVAPALOS MADON } \\
\text { ADOBE } 8 \times 8 \times 8 \\
\end{array}$ & & & & & & 25 & \\
\hline & \begin{tabular}{|c|} 
NAVAPALOS MARRON \\
BLOQUE \\
\\
$4 \times k \times k=4$
\end{tabular} & & & & & & 10.7 & \\
\hline & 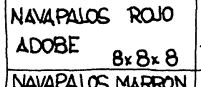 & & & & & & $|19.31|$ & \\
\hline & $\begin{array}{l}\text { NAVAPALOS MARRON } \\
\text { BREPAK } 95 \times 9,5 \times 95 \\
\end{array}$ & & & & & & 34.07 & \\
\hline & $\begin{array}{r}\text { MORTERO MAPDPON } \\
7 \times 7 \times 7\end{array}$ & & & & & & & 15.86 \\
\hline & $\begin{array}{r}\text { MORTERO RONO } \\
7 \times 7 \times 7 \\
\end{array}$ & & & & & & & 10.71 \\
\hline & 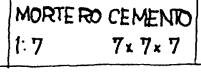 & & & & & & $\rightarrow$ & 77.41 \\
\hline
\end{tabular}

5. MAMPOSTERIA DE ADOBES: RESISTENCIAS A COMPRESION Y COMPRESION DIAGONAL (CORTANTE)

Siguiendo la norma peruana mencionada, se prepararon muretes para su ensayo a compresión (Fig. 2) y a cortante (Fig. 3).

En la Tabla 2, se recogen de forma esquemática las caracteristicas dimensionales de las probetas de ensayo, las distintas combinaciones entre los cuatro tipos de adobes ensayados y los tres tipos de material de unión, asi como los valores medios de los resultados de tres ensayos a compresión (valores situados en la parte superior izquierda) y a compresión diagonal (valores en la parte inferior derecha).

\section{Observaciones:}

- A compresión se observa una ligera mejoria en las muestras con juntas de mortero de cemento.

- A compresión diagonal la mayoria de las roturas se producen más por deslizamiento (Fig. 3) que por corte aunque aparecen algunos bloques rotos (Fig. 4). En cualquier caso la rotura es frágil.

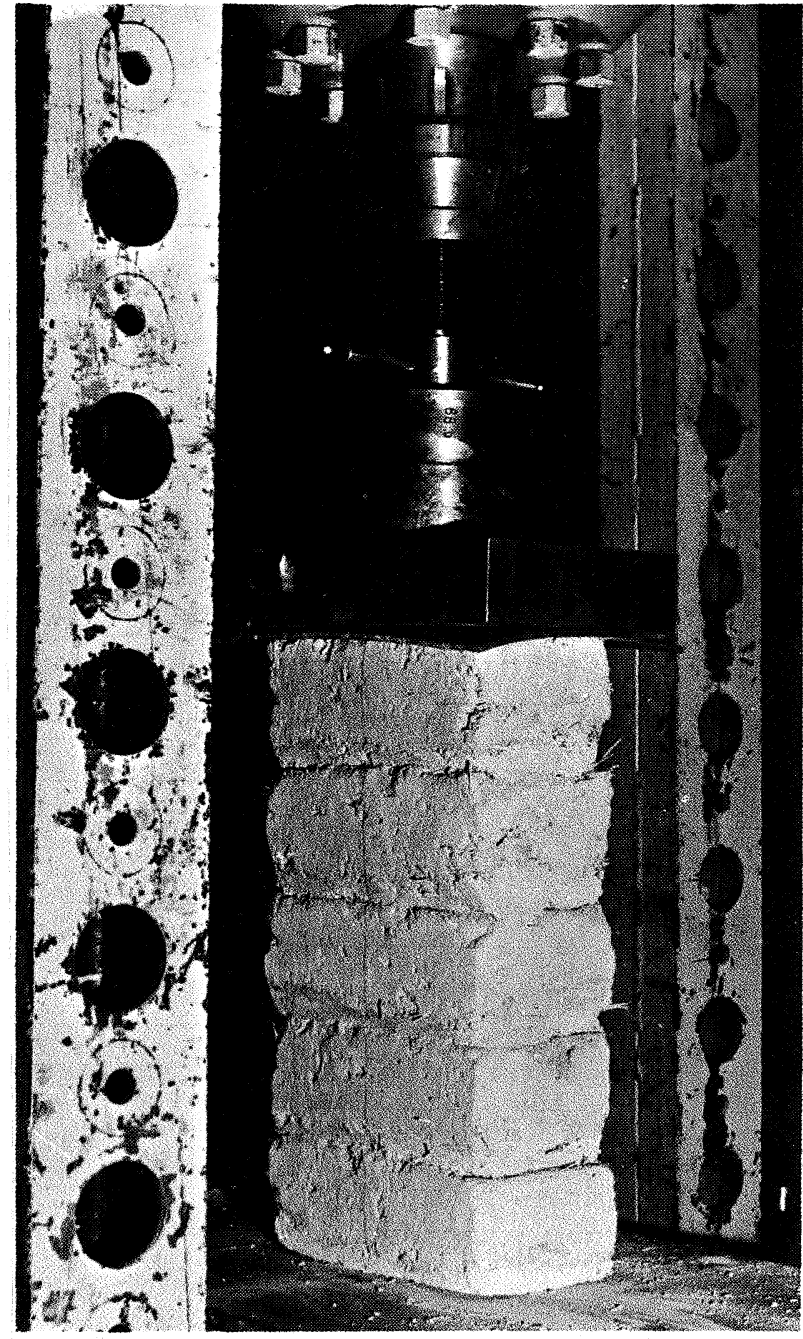

Fig. 2

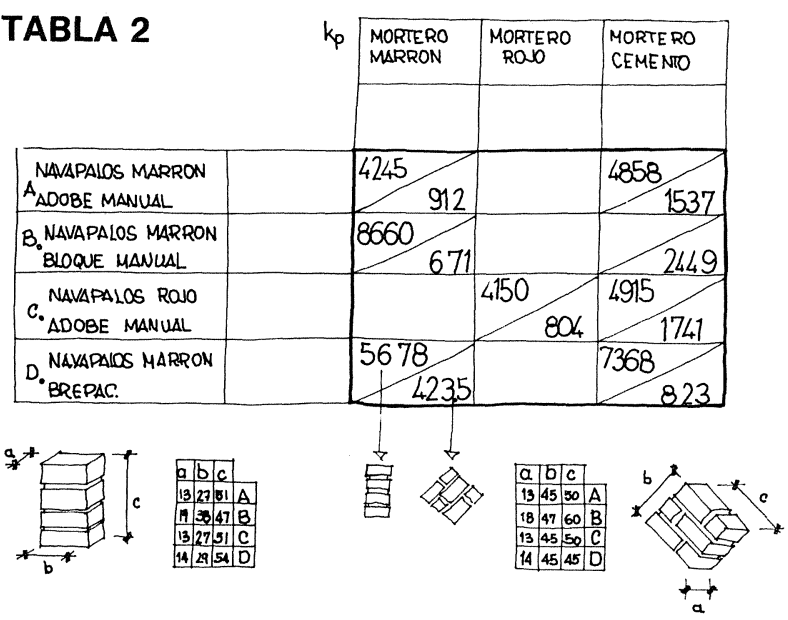

- Los valores obtenidos a compresión diagonal en las probetas Navapalos marrón Brepak, son singularmente bajos debido a la falta de adherencia entre las caras de los elementos y el mortero de unión, hasta el punto que algunas probetas rompieron durante su manipulación. 


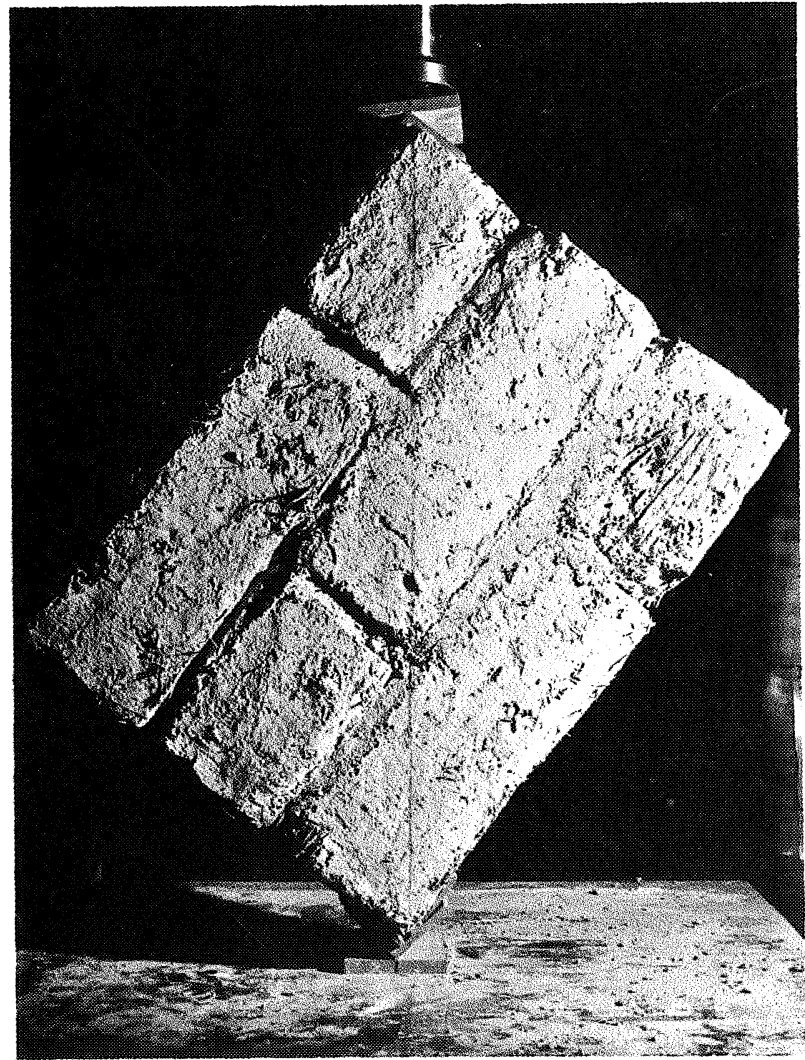

Fig. 3
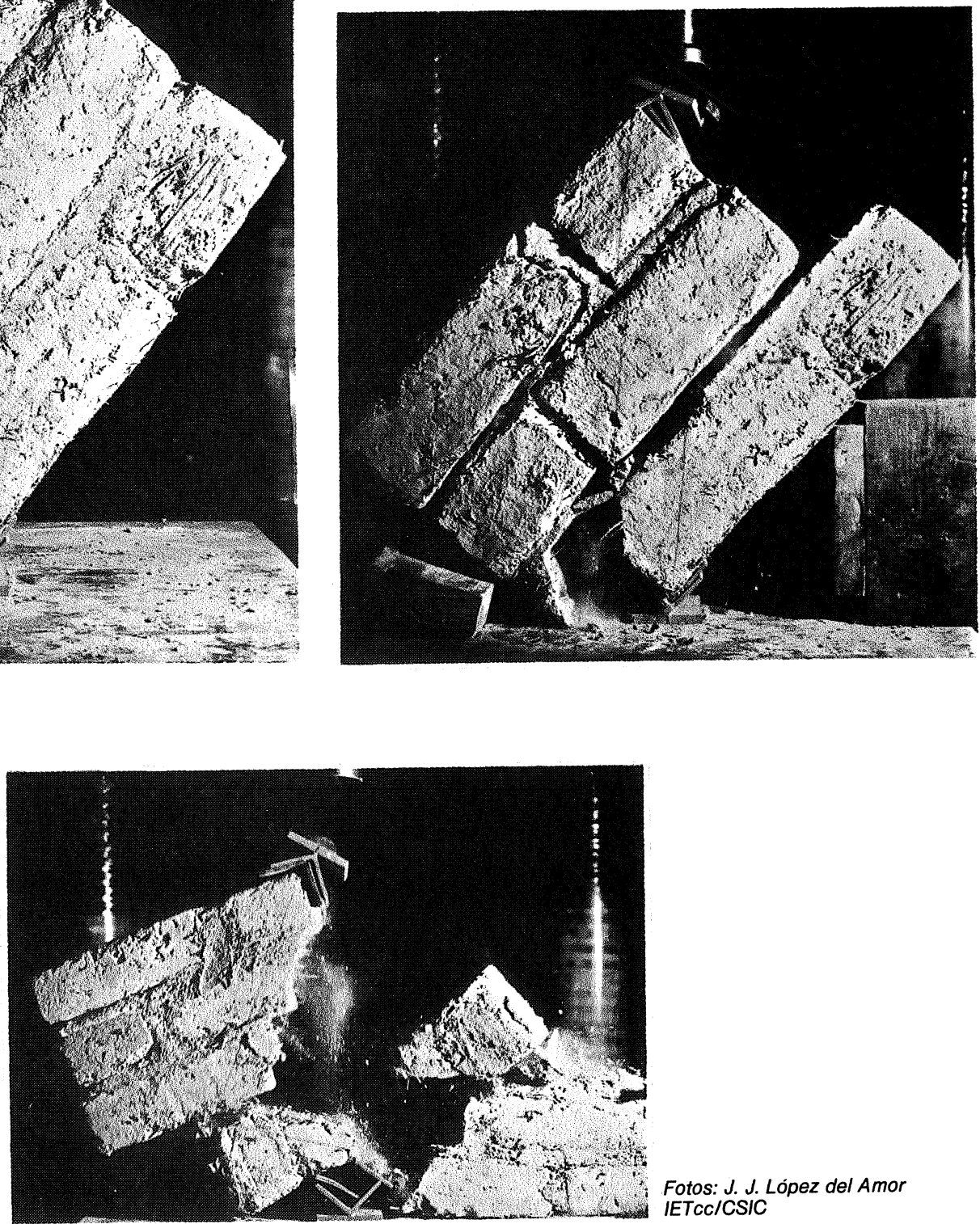

Fig. 4

\section{RESUMEN DE RESULTADOS}

Las Tablas 3 y 4, pretenden dar una información global de los resultados medios obtenidos en función de todas las variables manejadas. La información se ofrece en forma numérica y gráfica para una percepción más intuitiva.

Los valores de las tensiones de rotura a compresión diagonal se han obtenido a partir de la fórmula que figura en la norma peruana (2) según la cual $f_{t}=$ (esfuerzo último del murete de ensayo) $=P / 2 c a$. En donde $P$ es la carga de rotura (Tabla 2 ) y c y a son las dimensiones de la probeta de ensayo reseñadas en la misma Tabla.

La Tabla 5, a efectos comparativos, recoge los resultados de este trabajo asi como los obtenidos en las investigaciones mencionadas que se desarrollaron en México y Perú. 

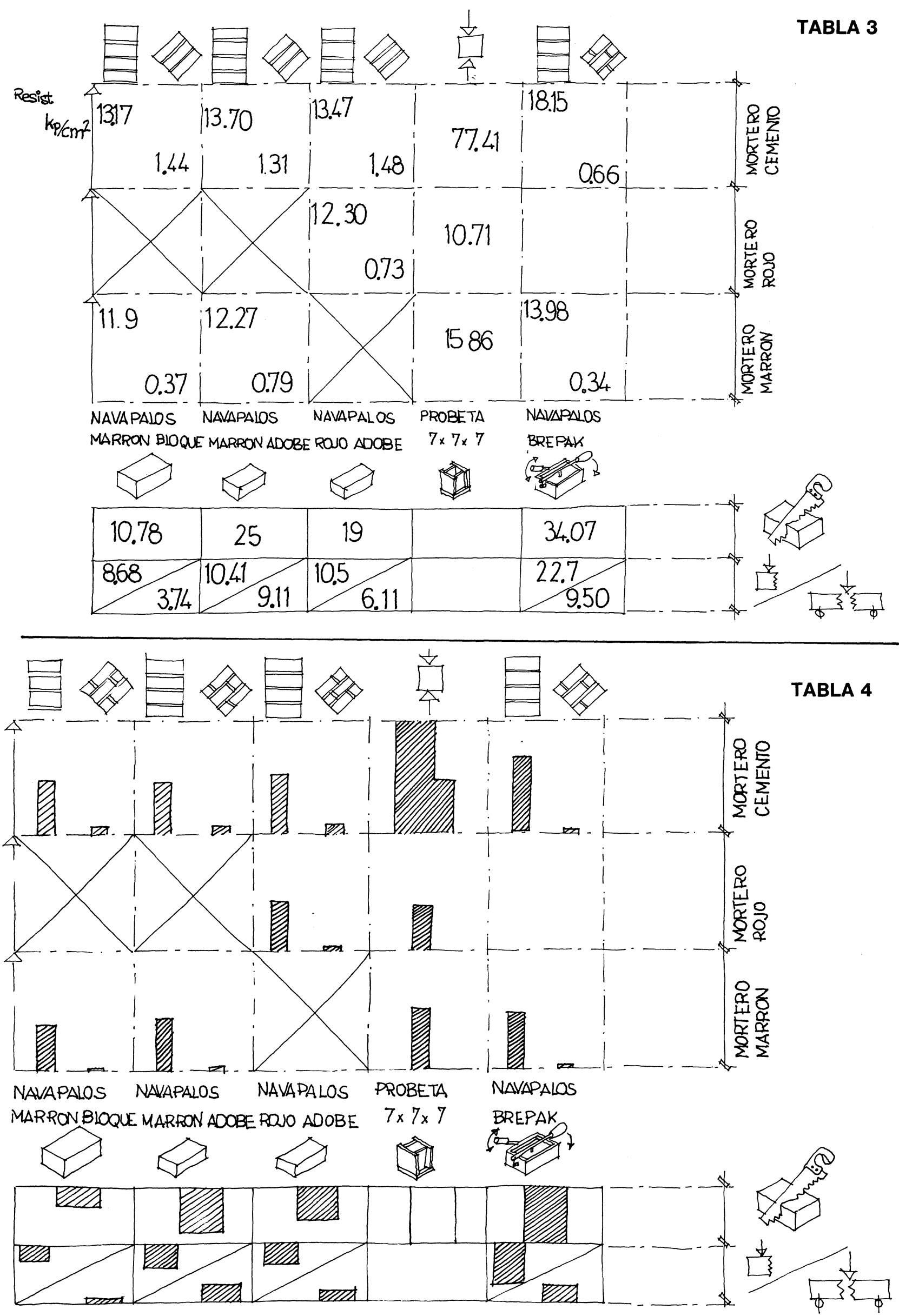
TABLA 5

\begin{tabular}{|c|c|c|c|c|}
\hline ESPECIMEN & TIPO DE ENSAYO & ESPAÑA & MEXICO & PERU \\
\hline $\begin{array}{l}\text { ADOBE } \\
\text { BLOQUE }\end{array}$ & $\begin{array}{r}\text { COMPRESION } \\
k_{\mathrm{Pkm^{2 }}}\end{array}$ & $\begin{array}{c}10.7 \text { a } 3407^{*} \\
18.33(22.27)^{*}\end{array}$ & $\begin{array}{c}5.2 a 16 \\
10 \\
\end{array}$ & $\begin{array}{c}11 \text { a } 17 \\
14\end{array}$ \\
\hline $\begin{array}{l}A D O B E \\
\text { BLQUE }\end{array}$ & $\begin{array}{r}\text { FLEXO TRACCION } \\
k_{\mathrm{P} / \mathrm{cm}^{2}}\end{array}$ & $\begin{array}{c}37 \% \text { a } 9.50 \\
7.11\end{array}$ & $\begin{array}{c}2 \times 4 \\
3\end{array}$ & $\begin{array}{c}3 a 8 \\
5 \\
\end{array}$ \\
\hline PILAS & $\begin{array}{r}\text { COMPRESION } \\
\mathrm{kP}_{\mathrm{P} / \mathrm{cm}^{2}}\end{array}$ & $\begin{array}{c}11.81 \text { a } 14 \\
12.93\end{array}$ & 13.4 & $\begin{array}{c}814 \\
\text { mes कño }\end{array}$ \\
\hline MUREIE & $\begin{array}{l}\text { COMPRESION } \\
\text { PIAGONAL } \mathrm{k}_{\mathrm{P} \mathrm{km}^{2}}\end{array}$ & $\begin{array}{c}0.73 \text { a } 1.48 \\
1.15\end{array}$ & 1.3 & 0.27 \\
\hline $\begin{array}{l}\text { DMENSION } \\
\text { MAS USUAL }\end{array}$ & & $27 \times 13 \times 10$ & $4 \times 16 \times 24$ & $8 \times 20 \times 10$ \\
\hline
\end{tabular}

"Incluye los valores de los adobes realizados con prensa “BRE PAK».

\section{OBSERVACIONES Y COMENTARIOS A LOS RESULTADOS}

Respecto a los resultados de este trabajo:

1. No se aprecian diferencias importantes en el comportamiento resistente de los diversos tipos de adobes, aunque si es considerable, si se comparan con los bloques de máquina.

2. Las juntas de mortero de cemento, para todos los tipos de adobe, suponen incrementos del orden del $10 \%$, en los ensayos de probetas a compresión (ver Tabla 3).

3. No se aprecian diferencias importantes entre los diversos tipos de adobes y bloques para un mismo tipo de junta. Por contra, el comportamiento a compresión diagonal mejora notablemente para el caso de juntas con mortero de cemento, con incrementos de más del $100 \%$, respecto de las realizadas con material de unión a base de tierra.

4. Como elementos aislados, el que presenta un comportamiento más uniforme y da las mayores prestaciones en todos los ensayos efectuados es el bloque marrón de máquina Brepak.

5. En el trabajo anteriormente citado (1) no se recomienda el ensayo de medios elementos a compresión, por considerar que los resultados se afectan considerablemente por el excesivo confinamiento lateral producido por las placas de carga. En este trabajo se ha obviado este punto, debido a las diferentes medidas de las piezas españolas frente a las mexicanas y peruanas, que los hacen más esbeltos $(24 \times 16 \times 4$ y $40 \times 20 \times 8)$ resultando el medio elemento de este trabajo muy próximo a las probetas cúbicas

recomendadas por peruanos y mexicanos.

En cuanto a los resultados obtenidos comparativamente con los aportados por mexicanos y peruanos, se desprenden las siguientes conclusiones:

a) Las resistencias a compresión de los elementos realizados manualmente son muy semejantes en los tres países, algo mayores los de este trabajo.

b) Las resistencias de tracción por flexión conseguidas, son bastante parecidas a las obtenidas en Perú y muy superiores a las mexicanas.

c) Mientras que en los valores de México la resistencia de los elementos es sensiblemente igual a la de las mamposterias a compresión, Perú consigue esta igualdad para probetas con un año de edad, siendo la relación de resistencias en el momento del ensayo del orden del $40 \%$, relación muy próxima a la obtenida en los ensayos de este trabajo.

d) En todos los casos la resistencia al corte no depende sustancialmente de las dimensiones del bloque.

e) La resistencia al corte depende fundamentalmente del grado de monolitismo conseguido por el material de junta. (Téngase presente que ni Perú ni México utilizan mortero de cemento y arena en sus ensayos).

f) En resumen, los resultados conseguidos se aproximan a los de México al utilizar juntas de mortero de cemento y arena y presentan valores intermedios entre los dos trabajos comparados, cuando se utilizan juntas de mortero de tierra.

\section{BIBLIOGRAFIA}

(1) OTTAZI PASINO, G. y VARGAS NEUMAN, J.: «Investigación comparativa sobre la resistencia del adobe». Departamento de Ingeniería de la Pontificia Universidad Católica del Perú.

(2) ININVI, INSTITUTO NACIONAL DE INVESTIGACION Y NORMALIZACION DE LA VIVIENDA DE PERU: "Construcciones en adobe. Disposiciones Especiales para Diseño Sismorresistente, NTE E 080 Adobe".

(3) GUINEA, M.a Jesús: "Normas relacionadas con construcciones de tierra". Informes de la Construcción, Vol. 37, n.o 377, pág. 69. Madrid, enerofebrero, 1986. 